\title{
Aesthetics, Digital Technology and Collaboration
}

\author{
Tommaso Colombino, Antonietta Grasso, David Martin, Jacki O'Neill ${ }^{1}$ \\ John Bowers ${ }^{2}$ \\ ${ }^{1}$ XRCE, \{firstname.surname@xrce.xerox.com\} \\ ${ }^{2}$ John Bowers Goldsmiths University of London, john.m.bowers@ gmail.com
}

\begin{abstract}
The workshop examines aesthetics-in-action through naturalistic studies focusing on the role of technology in artistic composition-production, performance, consumption, aimed at creating a body of knowledge to inform innovative technology design.
\end{abstract}

\section{INTRODUCTION}

As technology's reach extends into all aspects of our lives, art and technology become increasingly intertwined. Technology ranges from being implemented in - to integral to - the creation, exhibition, performance and appreciation of much art and aesthetic work. For example, graphic design is now predominantly achieved using applications such as Adobe Premier, Photoshop, Quark Express and so on; photography and photographic editing and manipulation is primarily digital, as is much film; many musicians use technologies for producing and performing music such as samplers, synthesisers and production applications. Artists are increasingly exploiting and exploring novel uses of digital technology. In all of these cases the properties - facilities, affordances, features, constraints - of the technology will necessarily impact, in some manner on the aesthetic product. We are aware that this impact can be seen in different lights. For example, there are movements that seek to critique mainstream technology through aesthetic subversion (such as the steampunk movement in modern music/culture). Interestingly, such movements point to the limits of digital technologies in the arts.

There have been many ethnographies of work and the way technologies are employed in these settings technologies - their capacities and effects in practice. There is also a long history of academic work on aesthetics in a range of disciplines - art and design, critical theory, sociology and psychology. However, we find that there seems to be a relative lack of social studies of aesthetics-in-action. That is, with aesthetics as visible, available, social practice in artistic composition-production, performance or consumption, (what might be called the aesthetic life-cycle). How do we understand technologies and artistic endeavour, performance and aesthetic experience? How can this be used to evaluate existing technologies and inform the design of technologies to support existing or enable new ways of producing and appreciating artistic work? What is the situated, practical, social and cultural nature of aesthetics?

A response to a piece of music under composition or the manufacture of an aesthetic object or an appreciation of a painting is clearly tied to an engagement in a place in a time. Likes and dislikes, notions of beauty, colour preferences, ideas about harmony and so forth are fundamentally social, cultural and practical and it is important to try and understand these phenomena. Different encounters produce different experiences as the context provided by and brought to the aesthetic object changes. For example, graphic designers use talk, along with technologies and design work as a way of exploring, translating, realising and sharing a design - what it is the customer wants, might like and so on. Talk also serves like a gesture in an evaluation or an appreciation; the words fix their meaning as part of an activity in relation to a design. Just what a 'rich, luxurious red' means is elaborated by and elaborates a specific colour palette for a certain product for a particular customer and so forth. This discourse ties the design's aesthetics to the brief (or 'requirements') and forms the basis for later activities like the presentation (performance) of the designs to the customer. Also of interest may be the role of 'supplementary materials' such as catalogues, programme notes, etc. and critical aesthetic discourse itself as means for configuring people's practical understanding and appreciation of works.

\section{THE TOPICS}

When we consider the role of technology in this we can see that it impacts on the production, performance and consumption of many types of art. It facilitates, scaffolds and often enables many different possibilities to be tried and in doing so it changes both the manner of aesthetic production and what can be produced. We are interested in social studies relating to the composition-production, performance and consumption of artwork, and crucially the role of technology in this. We are particularly interested in ethnographies that capture and explicate the following in natural settings, in action:

\footnotetext{
* visual arts and design

* the production and appreciation of colour, structure and harmony

* visual and visible aspects of performance

* but... we are also interested in material from other arts disciplines - e.g. music, drama, performance arts

* aesthetics in action and practice

* aesthetics work and its relation to different purposes in different settings, e.g. the interrelation of the work of production to performance

* aesthetics talk and the work it does in production, performance and composition
}

By focusing on what we think is an under-explored, but technologically interesting, area of research - that is the natural practices of aesthetic 'work' - we aim to bring together a set of 
practitioners/researchers and their data, as a first step in creating a body of knowledge on aesthetics in action. This can provide a sound basis for understanding how technology use impacts art. Such studies have previously proved effective, in for example the workplace, in informing the design of innovative new technologies based on actual working practices. We would hope to convene a further workshop on the design of technologies for aesthetic 'work' which could build on this body of knowledge.

\section{WORKSHOP PROCEDURE}

We welcome work in many stages, as well as mature work we are interested in seeing work-in-progress and raw(ish) data as the workshop will provide an opportunity to share ideas and do joint analysis. The workshop will begin with short introductory presentations from the organisers on their work on 1) the visual arts - studies of graphic designers, including their use of technology to create their designs - and 2) music - studies of the organisation of improvised electro-acoustic music and the construction of technologies by artists for making such music. The main body of the workshop will consist of short presentations from the participants, with the possibility of a data session where we can spend time analysing data extracts (transcripts, video, etc.). Papers will be organised into sessions to provoke exploration around similar topics, technologies, settings and time will be provided at the end of each session to enable discussion, debate etc. In addition, we are researching the possibility of bringing in an artists/musician to perform/demonstrate and assist in the workshop (art-in-action, as it were). The day will finish with a synthesis session, during which the workshop poster will be constructed and next steps. 